\title{
Histological study of postnatal development of mouse tongues
}

\author{
YIFENG JIANG ${ }^{1}$, ZHEN DU $^{2}$ and LONG CHEN $^{2}$ \\ ${ }^{1}$ Department of Stomatology, Shandong Medical College, Linyi, Shangdong 276002; \\ ${ }^{2}$ Department of Stomatology, Linyi People's Hospital, Linyi, Shandong 276000, P.R. China
}

Received April 12, 2017; Accepted September 8, 2017

DOI: 10.3892/etm.2017.5350

\begin{abstract}
Numerous factors, including trauma, tumors and myophagism, may lead to tongue defects, which are mostly repaired via muscular flaps. However, these methods cannot restore the muscular function and gustation function of the tongue. Intensive research on tongue development may offer useful clues for tongue regeneration based on tissue engineering or stem cell therapy. In the present study, staining results revealed that tongue muscle fibers became larger, mature and stronger, and the foliate and fungiform papillae also became mature from newborn to adult C57BL/6J genetic background mice. Immunofluorescence staining and polymerase chain reaction results revealed that $\mathrm{C}$-kit was dynamically expressed in muscle cells, as well as in foliate and fungiform papilla cells from newborn to adult stages. The expression level decreased from P1 to P15 and increased at P90. The immunofluorescence staining results revealed that $\mathrm{Ki}-67$ was expressed in muscle cells and papilla cells from newborn to adult stages, and high expression was observed at P6 and P15. In addition, the immunofluorescence staining results also demonstrated that msh homeobox 2 (Msx2) was dynamically expressed in postnatal tongue muscle cells; however, almost no expression was detected in papilla cells. There was relative high expression level of Msx 2 at P1 and P6 stages, but this gradually decreased from P15, and it was expressed primarily in the muscle cells located in the marginal zone of the tongue at P90. These findings suggest that the amount of c-kit-expressing precursor cells in tongue muscle and papilla cells increases to promote tongue development at the early postnatal stage and to maintain homeostasis and functional adaptation of the tongue in the adult stage. Furthermore, Msx 2 may serve an important role in postnatal tongue muscle development. The present study also suggests that C-kit and Msx 2 may be used as cell markers for postnatal tongue regeneration and self-repair, and may provide an approach for developing treatment methods for tongue diseases with a postnatal onset.
\end{abstract}

Correspondence to: $\mathrm{Mr}$. Long Chen, Department of Stomatology, Linyi People's Hospital, 27 East Jiefang Road, Linyi, Shandong 276000, P.R. China

E-mail: ok365@yeah.net

Key words: postnatal development, mice, tongue, histological study

\section{Introduction}

Normal tongue development has a crucial role in craniofacial development, particularly for jaw and palate development (1). Tongue primordium is comprised of mesenchyme cells derived from cranial neural crest cells (CNCCs), ectoderm-derived lingual epithelium in the distal portion and the endoderm-derived proximal portion (2). The myoblasts migrated from the occipital somites surround the CNCCs during the process of tongue development (2). Core myogenic regulators, such as MyoD, myogenic factor (Myf)5 and Myf6 (also known as MRF4) have important roles in tongue muscle development (3). However, the tongue muscle also exhibits unique characteristics that are distinct from those of other skeletal muscles, and different regulatory mechanisms may be in place. Previous studies demonstrated that transforming growth factor $\beta 1$, Smad4 and fibroblast growth factor 6 signaling pathways regulate myogenic differentiation and myoblast fusion during tongue development $(2,3)$. It should be noted that most previous studies focused on embryonic development of mice tongues, whereas the postnatal development of the tongue has remained to be fully explored. However, the onset of numerous tongue diseases, such as geographic tongue and fissured tongue, have a postnatal onset, and numerous factors, including trauma and tumors, may lead to postnatal tongue defects (4). Thus, intensive research on tongue development may offer useful clues for better understanding the possible mechanisms of tongue disease, and provide novel approaches for tongue regeneration. The present study performed a histological analysis of the postnatal development of mouse tongues with the aim of identifying possible regulatory mechanisms underlying postnatal tongue development.

\section{Materials and methods}

Animals. C57BL/6J genetic background mice were obtained from Shanghai SIPPR-Bk Lab Animal Co., Ltd. (Shanghai, China). A total of 3 maternal mice (12 weeks old; 22-24 g) were used to obtain newborn mice in the present study. All experimental animal protocols were reviewed and approved by the Ethics Committee of Linyi People's Hospital (Linyi, China). Mice lived in a specific pathogen-free animal room with a temperature of $22^{\circ} \mathrm{C}$, and a 12 -h light/dark cycle. The development time of the mouse embryos was calculated with the day of vaginal plug detection designated as E0.5. At every time-point, the nodes of fifteen tongues were harvested 
from immature mice, and five tongues from P90 mice were obtained and fixed with $4 \%$ paraformaldehyde overnight at $4^{\circ} \mathrm{C}$, followed by washing with PBS. Subsequently, these tissues were embedded in paraffin and sectioned at a thickness of $5 \mu \mathrm{m}$ for the subsequent experiments.

Hematoxylin and eosin $(H \& E)$ staining. Tongue sections of $\mathrm{P} 1, \mathrm{P} 3, \mathrm{P} 6, \mathrm{P} 10, \mathrm{P} 15$ and $\mathrm{P} 90$ mice were deparaffinized at $60^{\circ} \mathrm{C}$ for $2 \mathrm{~h}$, followed by rehydration in a graded series of ethanol and washing briefly in distilled water. Sections were stained using the regular H\&E staining method at room temperature. Briefly, sections were stained with Harris hematoxylin solution for $8 \mathrm{~min}$ and eosin-phloxine solution for $1 \mathrm{~min}$. Slices were cleared in xylene and mounted with resinous mounting medium to be observed and under a microscope, where images were captured.

Immunohistochemical staining. Tongue slices of P1, P3, P6, P10, P15 and P90 mice were dewaxed and immunohistochemical staining was performed according to the following procedures: First, the sections were blocked using $3 \%$ bovine serum albumin (Sigma-Aldrich; Merck KGaA, Darmstadt, Germany) in PBS for $40 \mathrm{~min}$. Subsequently, the sections were incubated with the respective primary antibody to Ki67 (ab15580; Abcam, Cambridge, MA, USA; 1:150) or C-kit (ab5506; Abcam; 1:150) overnight at $4^{\circ} \mathrm{C}$. The slices were then washed with PBS and incubated with horseradish peroxidase-labeled secondary antibody (KIT-5905; Maixin, Fuzhou, China; 1:250) at room temperature for $60 \mathrm{~min}$, followed by development using a diaminobenzidine staining kit (KIT-5905; Maixin, Fuzhou, China) for 3-5 min at room temperature. Finally, the slices were counterstained with hematoxylin at room temperature for $1 \mathrm{~min}$, and mounted with resinous mounting medium for observation and capturing of images.

Immunofluorescence staining. Slices of tongue samples of P1, P3, P6, P10, P15 and P90 mice were prepared as described above. Then sections were incubated with primary antibody to msh homeobox 2 (Msx2; ab223692; Abcam; 1:150 dilution) overnight at $4^{\circ} \mathrm{C}$. Subsequently, the slices were washed with PBS and incubated with secondary antibody (Alexa Fluor 488-conjugated donkey anti-rabbit; A-21206; Invitrogen; Thermo Fisher Scientific, Inc., Waltham, MA, USA) at a dilution of $1: 500$ for $45 \mathrm{~min}$ at $37^{\circ} \mathrm{C}$, and were counterstained with DAPI. Finally, the slices were mounted with glycerinum mounting medium for observation and image capturing under a fluorescence microscope.

Reverse-transcription quantitative polymerase chain reaction (RT-qPCR). For RT-qPCR analysis, the anterior mouse tongues were dissected and the total RNA was isolated using TRIzol reagent (Invitrogen; Thermo Fisher Scientific, Inc.). The total RNA was converted to complementary DNA according to the instructions of the Prime Script-RT reagent kit (RR037A; Takara Bio, Inc., Otsu, Japan). qPCR was performed using the SYBR-Green system (DRR041A; Takara Bio, Inc.) in a total volume of $20 \mu \mathrm{l}$ with the MyiQ Single-Color Real-Time PCR Detection System (Bio-Rad Laboratories, Inc., Hercules, CA, USA). The expression of c-kit relative to $\beta$-actin and calculated using the $2^{-\Delta \Delta \mathrm{Cq}}$ method (5) with normalization to the expression levels at P15. Each $\mathrm{qPCR}$ reaction was repeated three times. The primers for c-kit and $\beta$-actin were identical to those used in a previous study (6).

Statistical analysis. Values are presented as the mean \pm standard deviation. The statistical analysis of the qPCR results was performed using SPSS v.16 software (SPSS, Inc., Chicago, IL, USA). Analysis of variance followed by a post-hoc multiple comparisons test (Duncan's test) was used to compare the results between different groups. $\mathrm{P}<0.05$ was considered to indicate a statistically significant difference.

\section{Results}

Histological structures of postnatal mouse tongues. The $\mathrm{H} \& \mathrm{E}$ staining results indicated that the tongue muscle fibers of the mice became larger, mature and stronger during the development from newborn to adult, and an increased amount of adipose tissues surrounded the tongue muscle in adult mice. The foliate and fungiform papillae also became mature, but the foliate papillae gradually decreased from newborn to adult mice (Fig. 1).

Expression of $\mathrm{C}$-kit and $\mathrm{Ki}$-67 in postnatal mouse tongues. Immunohistochemical staining results demonstrated that C-kit, a marker of mesenchymal progenitor cells, was expressed in muscle cells as well as in foliate and fungiform papilla cells. The expression was weaker in foliate papilla and fungiform papilla cells when compared with that in muscle cells. The expression of C-kit in muscle and papilla cells was gradually downregulated from the newborn to the adult stage, but was markedly increased at P90 (Fig. 2). The qPCR results also demonstrated that c-kit mRNA levels displayed dynamic changes during postnatal development of the tongue, as they were decreased from P1 to P15, but then increased at P90 (Fig. 3). Duncan's multiple range test indicated that the expression levels of c-kit exhibited no significant difference between the P1 and P3 stage, or between the P6 and P10 stage, whereas they differed significantly between the any other two stages $(\mathrm{P}<0.05$; Fig. 3$)$. These results implied that at the early postnatal stage, c-kit expression and the amount of c-kit-expressing cells was higher to promote tongue development, and in the adult stage c-kit was expressed to maintain the homeostasis and functional adaptation of the tongue.

The immunohistochemical staining results also indicated that $\mathrm{Ki}-67$, a marker of cell proliferation, was expressed in muscle cells and papilla cells from the newborn to the adult stage, with relatively higher expression level at P6 and P15, suggesting increased cell proliferation at this stage (Fig. 4).

Expression of Msx2 in postnatal mouse tongues. Msx2, a homeobox transcription factor, has a crucial role in vascular smooth muscle and skeletal muscle development. The present study aimed to explore the expression of Msx2 in the tongue muscle. The immunofluorescence staining results indicated that Msx 2 was expressed in postnatal tongue muscle cells, but almost no expression was present in papilla cells. In addition, 


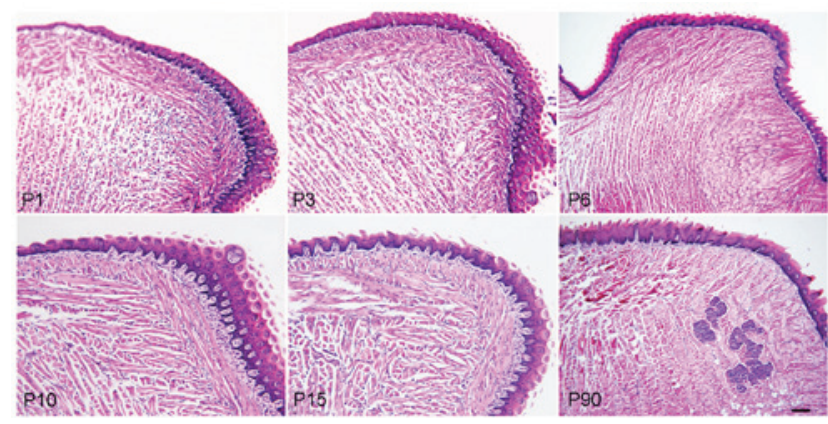

Figure 1. Hematoxylin and eosin staining of mouse tongues at various postnatal development stages. Tongue muscle fibers became larger, mature and stronger from newborn (P1) to adult (P90) stage, and an increased amount of adipose tissues surrounded the tongue muscle in adult mice. The foliate and fungiform papillae became mature, but the foliate papillae gradually decreased from P1 to P90. Scale bar, $100 \mu \mathrm{m}$. P, postnatal.

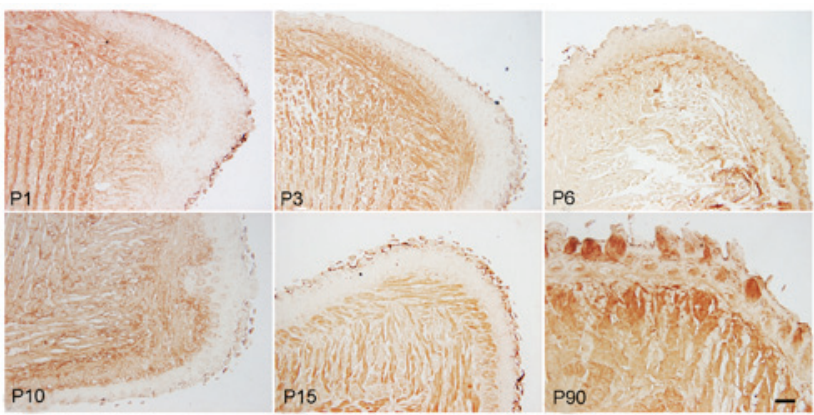

Figure 2. Immunohistochemical staining results for C-kit in mouse tongues at various postnatal stages. C-kit was expressed in the muscle cells, as well as foliate and fungiform papilla cells, and the expression was gradually downregulated from P1 to P15, and then increased at P90. Scale bar, $100 \mu \mathrm{m}$. $\mathrm{P}$, postnatal.

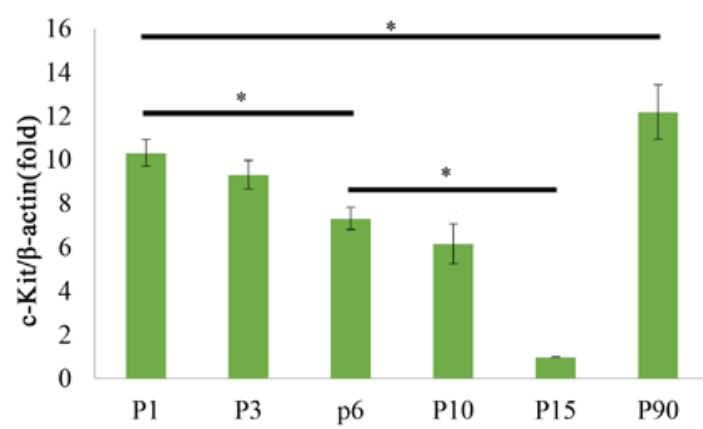

Figure 3. c-kit mRNA expression levels in mouse tongues at various postnatal development stages determined by reverse-transcription quantitative polymerase chain reaction analysis. c-kit mRNA expression levels decreased from $\mathrm{P} 1$ to $\mathrm{P} 15$, and then increased at $\mathrm{P} 90 .{ }^{*} \mathrm{P}<0.05$. $\mathrm{P}$, postnatal.

there was relatively high expression level at the P1 to P6 stage, which gradually decreased from P15 (Fig. 5).

\section{Discussion}

During the embryonic development of the mammalian tongue, early tongue movement is adapted for certain functional activities, including suckling, swallowing and chewing. During postnatal stages, the tongue also continually develops, the

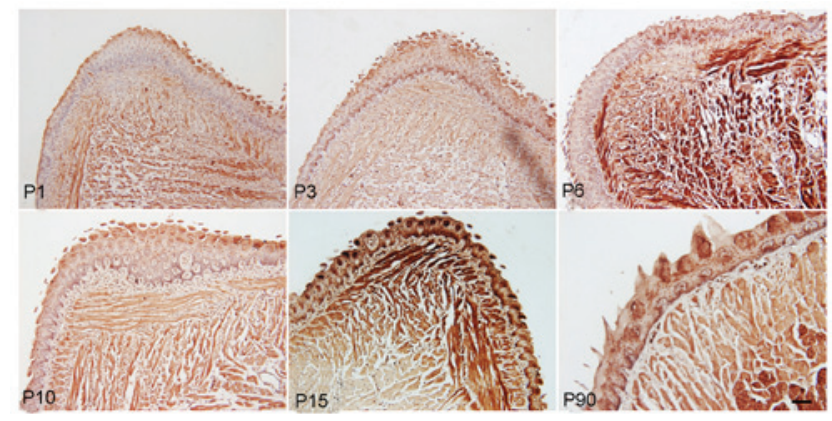

Figure 4. Immunohistochemical staining results for Ki-67 in mouse tongues at various postnatal development stages. Ki-67 was expressed in muscle cells and papilla cells, and exhibited relatively high expression at P6 and P15 stage. Scale bar, $100 \mu \mathrm{m}$. P, postnatal.

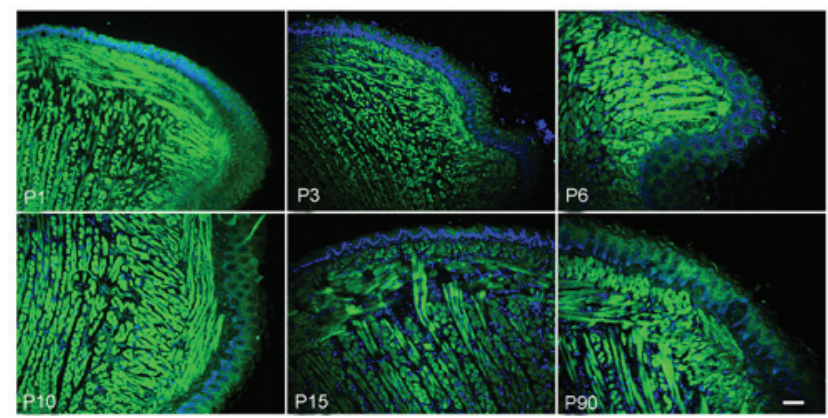

Figure 5. Immunofluorescence staining results for Msx 2 in mouse tongues at various postnatal development stages. Msx 2 was strongly expressed in postnatal tongue muscle cells, but almost no expression was observed in papilla cells from P1 to P90, Expression was relatively high at P1 and P6; however, it gradually decreased from P15 and was mainly expressed in the muscle cells in the marginal zone of the tongue at P90. Scale bar, $100 \mu \mathrm{m}$. P, postnatal; Msx2, msh homeobox 2.

tongue size increases and the papilla become mature. However, the number, position and ratio of different types of papillae gradually changes to adopt different functions $(7,8)$. The results of the present study also indicated that tongue muscle fibers became larger, mature and stronger during development from newborn to adult mice. The foliate and fungiform papillae also became mature, but the former gradually decreased from the newborn to the adult stage, which may have been for the purpose of acquiring a gustation function as well as adapting to oral functions such as vocalization and eating.

Numerous factors, including trauma, myophagism and tumors, may lead to tongue defects. The implementation of various flaps, such as the forearm flap, are the major repair methods for tongue defects (9). However, these methods are not able to repair the tongue muscle and gustation function. Thus, intensive research into tongue development may offer useful clues for the development of novel tongue regeneration strategies based on tissue engineering and stem cell therapy. Previous studies have indicated that muscle regeneration after injury exhibits similarities to muscle development, and that quiescent myogenic cells, also called satellite cells, which are situated beneath the basal lamina that surrounds each myofiber, were activated to proliferate, differentiate and fuse to form multinucleated myofibers (10-12). Other types of non-muscle stem cells may also participate in this process. 
The results of the present study indicated that C-kit, a marker of mesenchymal progenitor cells $(13,14)$, and Ki-67, a marker of cell proliferation (15), were expressed in muscle cells, foliate papilla cells and fungiform papilla cells from mouse tongues from newborn to adult stages. It was implied that precursor cells may exist among tongue muscle and papilla cells that express c-kit to promote tongue muscle and papilla cell development or maintain the homeostasis and functional adaptation of the tongue. These findings also offered certain clues regarding c-kit being a potential target for tongue regeneration, self-repair or treatments for tongue diseases with a postnatal onset. In addition, the expression profile of $\mathrm{Ki}-67$ suggests that cell proliferation was increased at P6 and P15, indicating that taste bud development may be promoted during this stage.

The mechanisms underlying myogenesis in vertebrate organisms have been extensively investigated, and numerous factors, including interleukin (IL)-4, IL-6, MyoD, Myf5, Pax3 and Pax-7, have important roles in muscle development and tongue muscle satellite cell activity (16-18). However, the exact molecular mechanisms of muscle remodeling have remained elusive. During the development of muscle, the Msx family of transcription factors also has crucial roles. A previous study has indicated that Msx1 and histone H1b cooperate to inhibit muscle differentiation in cell culture and in Xenopus ectodermal explants (19). Overexpression of LIM homeobox 2 (Lhx2) completely inhibited the myotube-forming capacity of $\mathrm{C} 2 \mathrm{C} 12$ cells and primary myoblasts, and the muscle dedifferentiation factors Msx 1 and Msx2 were strongly induced by the Lhx2 overexpression or inhibited by decreased Lhx2 expression (20). The results of the present study indicated that Msx2 was highly expressed in tongue muscle cells during postnatal development, but almost no expression was detected in papilla cells. These results implied that Msx2 may have an important role in the postnatal development of tongue muscles, and may also be a marker for tongue muscle regeneration and self-repair or for tongue muscle diseases with a postnatal onset.

In conclusion, the present study indicated that muscle fibers of mouse tongues became larger, mature and stronger, and the foliate and fungiform papillae also became mature during development from newborn to adult. C-kit was dynamically expressed in muscle cells as well as in foliate and fungiform papilla cells from newborn to adult stages, suggesting that the number of c-kit-expressing cells increased to promote tongue development at the early postnatal stage and to maintain homeostasis and functional adaptation of the tongue in the adult stage. Ki-67 was also dynamically expressed in muscle cells, as well as in foliate and fungiform papilla cells, from newborn to adult stages, with high expression 1 at P6 and P15, which suggests increased cell proliferation at this stage In addition, Msx 2 was also dynamically expressed in postnatal tongue muscle cells, but almost no expression was detected in papilla cells. These results implied the presence of precursor cells among tongue muscle and papilla cells, and Msx2 may have an important role in postnatal tongue muscle development. The present study also suggests that c-kit and Msx2 may be cell markers for postnatal tongue regeneration and self-repair, and may provide an approach for developing treatment methods for tongue diseases with a postnatal onset.

\section{References}

1. Cong W, Liu B, Liu S, Sun M, Liu H, Yang Y, Wang R and Xiao J: Implications of the Wnt5a/CaMKII pathway in retinoic acid-induced myogenic tongue abnormalities of developing mice. Sci Rep 4: 6082, 2014

2. Iwata J, Suzuki A, Pelikan RC, Ho TV and Chai Y: Noncanonical transforming growth factor $\beta$ (TGF $\beta$ ) signaling in cranial neural crest cells causes tongue muscle developmental defects. J Biol Chem 288: 29760-29770, 2013.

3. Shuler CF and Dalrymple KR: Molecular regulation of tongue and craniofacial muscle differentiation. Crit Rev Oral Biol Med 12: 3-17, 2001.

4. Maciejewski A, Szymczyk C and Wierzgoń J: Triple skin island fibula free flap: A good choice for combined mandible and tongue defect reconstruction. J Reconstr Microsurg 24: 461-468, 2008.

5. Livak KJ and Schmittgen TD: Analysis of relative gene expression data using real-time quantitative PCR and the 2 (-Delta Delta C(T)) method. Methods 25: 402-408, 2001.

6. Wang X, Qi S, Wang J, Xia D, Qin L, Zheng Z, Wang L, Zhang C, Jin L, Ding G, et al: Spatial and temporal expression of c-Kit in the development of the murine submandibular gland. J Mol Histol 45: 381-389, 2014.

7. Miller IJ Jr and Smith DV: Proliferation of taste buds in the foliate and vallate papillae of postnatal hamsters. Growth Dev Aging 52: 123-131, 1988.

8. Liu HX, Ermilov A, Grachtchouk M, Li L, Gumucio DL, Dlugosz AA and Mistretta CM: Multiple shh signaling centers participate in fungiform papilla and taste bud formation and maintenance. Dev Biol 382: 82-97, 2013.

9. Song XM, Ye JH, Yuan Y, Zhang SY, Jiang HB and Wu YN: Radial forearm free flap for reconstruction of a large defect after radical ablation of carcinoma of the tongue and floor of the mouth: Some new modifications. ORL J Otorhinolaryngol Relat Spec 72: 106-112, 2010.

10. Hindi SM and Kumar A: TRAF6 regulates satellite stem cell self-renewal and function during regenerative myogenesis. J Clin Invest 126: 151-168, 2016.

11. Collins CA, Olsen I,Zammit PS, Heslop L, Petrie A, Partridge TA and Morgan JE: Stem cell function, self-renewal, and behavioral heterogeneity of cells from the adult muscle satellite cell niche. Cell 122: 289-301, 2005.

12. Wagers AJ and Conboy IM: Cellular and molecular signatures of muscle regeneration: Current concepts and controversies in adult myogenesis. Cell 122: 659-667, 2005.

13. Noda S, Horiguchi K, Ichikawa $\mathrm{H}$ and Miyoshi H: Repopulating activity of ex vivo-expanded murine hematopoietic stem cells resides in the CD48-c-Kit+Sca-1+lineage marker- cell population. Stem Cells 26: 646-655, 2008.

14. Sandstedt J, Jonsson M, Dellgren G, Lindahl A, Jeppsson A and Asp J: Human C-kit+CD45- cardiac stem cells are heterogeneous and display both cardiac and endothelial commitment by single-cell qPCR analysis. Biochem Biophys Res Commun 443: 234-238, 2014.

15. Zhang YK, Han XY and Che ZY: Effects of buyang huanwu tang combined with bone marrow mesenchymal stem cell transplantation on the expression of VEGF and $\mathrm{Ki}-67$ in the brain tissue of the cerebral ischemia-reperfusion model rat. J Tradit Chin Med 30: 278-282, 2010.

16. Yamane A, Mayo M, Shuler C, Crowe D, Ohnuki Y, Dalrymple K and Saeki Y: Expression of myogenic regulatory factors during the development of mouse tongue striated muscle. Arch Oral Biol 45: 71-78, 2000.

17. Kawabe Y, Wang YX, McKinnell IW, Bedford MT and Rudnicki MA: Carm1 regulates Pax7 transcriptional activity through MLL1/2 recruitment during asymmetric satellite stem cell divisions. Cell Stem Cell 11: 333-345, 2012.

18. Kirkpatrick LJ, Allouh MZ, Nightingale CN, Devon HG, Yablonka-Reuveni Z and Rosser BW: Pax7 shows higher satellite cell frequencies and concentrations within intrafusal fibers of muscle spindles. J Histochem Cytochem 56: 831-840, 2008.

19. Lee H, Habas R and Abate-Shen C: MSX1 cooperates with histone H1b for inhibition of transcription and myogenesis. Science 304: 1675-1678, 2004.

20. Kodaka Y, Tanaka K, Kitajima K, Tanegashima K, Matsuda R and Hara T: LIM homeobox transcription factor Lhx2 inhibits skeletal muscle differentiation in part via transcriptional activation of Msx1 and Msx2. Exp Cell Res 331: 309-319, 2015. 